\title{
Learning about non-predators and safe places: the forgotten elements of risk assessment
}

\author{
Maud C. O. Ferrari · Douglas P. Chivers
}

Received: 26 August 2010/Revised: 17 November 2010/Accepted: 19 November 2010/Published online: 4 January 2011

(C) The Author(s) 2010. This article is published with open access at Springerlink.com

\begin{abstract}
A fundamental prerequisite for prey to avoid being captured is the ability to distinguish dangerous stimuli such as predators and risky habitats from nondangerous stimuli such as non-predators and safe locations. Most research to date has focused on mechanisms allowing prey to learn to recognize risky stimuli. The paradox of learned predator recognition is that its remarkable efficiency leaves room for potentially costly mistakes if prey inadvertently learn to recognize non-predatory species as dangerous. Here, we pre-exposed embryonic woodfrogs, Rana sylvatica, to the odour of a tiger salamander, Ambystoma tigrinum, without risk reinforcement, and later try to teach the tadpoles to recognize the salamander, a redbellied newt Cynops pyrrhogaster-a closely related amphibian, or a goldfish, Carassius auratus, as a predator. Tadpoles were then tested for their responses to salamander, newt or fish odour. Pre-exposure to salamander did not affect the ability of tadpoles to learn to recognize goldfish as a predator. However, the embryonic pre-exposure to salamanders inhibited the subsequent learning of salamanders as a potential predator, through a mechanism known as latent inhibition. The embryonic pre-exposure also prevented the learned recognition of novel newts, indicating complete generalization of non-predator recognition. This pattern does not match that of generalization
\end{abstract}

M. C. O. Ferrari · D. P. Chivers

Department of Biology, University of Saskatchewan,

112 Science Place, Saskatoon, SK S7N 5E2, Canada

Present Address:

M. C. O. Ferrari ( $\square)$

Department of Environmental Science and Policy,

University of California, Davis, One Shields Avenue,

Davis, CA 95616, USA

e-mail: mcferrari@ucdavis.edu of predator recognition, whereby species learning to recognize a novel predator do respond, but not as strongly, to novel species closely related to the known predator. The current paper discusses the costs of making recognition mistakes within the context of generalization of predators and dangerous habitats versus generalization of non-predators and safe habitats and highlights the asymmetry in which amphibians incorporate information related to safe versus risky cues in their decision-making. Mechanisms such as latent inhibition allow a variety of prey species to collect information about non-threatening stimuli, as early as during their embryonic development, and to use this information later in life to infer the danger level associated with the stimuli.

Keywords Predator recognition - Non-predator recognition - Habitat learning - Latent inhibition . Embryonic learning · Decision-making · Information use

\section{Introduction}

Although costly, collecting information about their environment allows individuals to make better-informed decisions by decreasing the level of uncertainty associated with parameters such as local predation risk, patch profitability or mate quality (Dall et al. 2005). In the context of predation, the cost of making a mistake is often fatal; however, energy and time requirements prevent prey from living in completely secure microhabitats, as those conditions often impede on the ability of prey to obtain high-quality food or mates (Lima and Dill 1990; Lima 1998). As a result, prey are subject to strong selection pressure to obtain accurate information about the presence or absence of predators in their vicinity. However, information may be limited and we 
should expect a number of risk-aversive behaviours, which represent the default behavioural option to pursue in case of uncertainty (Sih 1992).

One of the most basic concepts related to adaptive antipredator responses is the ability of prey to distinguish between threatening stimuli such as predators or dangerous locations and non-threatening stimuli such as non-predators or safe locations (Brown and Chivers 2005). For a number of prey species, recognition of potential predators is mediated through experience. Prey may learn as a result of surviving their first encounter with a novel predatory species. Alternatively, prey may learn to recognize cues from predators using personal and/or social information (Dall et al. 2005). Numerous studies have reported the role of learning in predator recognition and a number of learning mechanisms have been documented, the most well-studied of all being through social learning (reviewed by Griffin 2004; Galef and Laland 2005) and through simultaneous pairing of novel predator cues and injured conspecific cues (reviewed by Ferrari et al. 2010), although the latter pertains mainly to aquatic species. A specific characteristic associated with both social learning of predators and conditioned learning with injured conspecific cues is the highly efficient one-step learning process, where a one-time conditioning is all that is needed for the learned association between risk and novel predator cues (e.g., sight, odour or sound) to be made (Curio 1988; Mathis et al. 1996; Wisenden and Harter 2001). The same one-time conditioning with alarm cues can teach prey the identity of a dangerous habitat. For example, fathead minnows (Pimephales promelas) can learn to recognize vegetated habitats or open water habitats as dangerous if alarm cues are paired with the habitat odours (Chivers and Smith 1995a, b). This rapid form of conditioning contrasts with the systematic improvement through trial-and-error process often documented in a foraging context. The efficiency of learned recognition of risk is somewhat paradoxical. Novel cues can quickly be associated with risk through a one-time learning event, but this mechanism leaves room for potential mistakes related to the association of risk with irrelevant novel cues. As a result, other mechanisms should be in place to decrease the likelihood of prey responding to extraneous stimuli.

Contrasting with the massive amount of information on learned recognition of risk, the literature on the role of learned recognition of non-risky (or safe) stimuli in shaping information use and decision-making by prey animals is almost non-existent. For some species, certain stimuli are innately perceived as non-threatening (see Griffin et al. 2001 for example). However, little is known about the extent of recognition of non-risky stimuli, particularly from a learning perspective. We make a clear distinction between not recognizing a cue as threatening and recognizing a cue as non-threatening. The former represent a neutral cue, which has yet to be classified as 'risky' or 'safe', while the latter represent a cue already classified as 'safe'. While classic behavioural assays cannot make the distinction between the two, this distinction is crucial in terms of information processing (see below). Latent inhibition and learned irrelevance (Acquistapace et al. 2003; Hazlett 2003) are modes of learning that label neutral stimuli encountered in the absence of negative reinforcement as 'safe' or 'irrelevant' and thus prevents a one-time learned association between these harmless stimuli and a potential risk cue. For example, Ferrari et al. (2006) showed that exposing fathead minnows to the odour of a novel trout without negative reinforcement once a day for six consecutive days prevented the formation of learned predator recognition of trout when minnows were subsequently exposed to injured conspecific cues paired with trout odour. Minnows exposed to a water control prior to the conditioning task successfully learned to recognize trout odour as a risky stimulus. While this mechanism is efficient, it requires prey to learn all neutral stimuli and label them as 'safe' to avoid mistakenly associating them with risky stimuli. A recent study has investigated ways in which prey species could get an edge on distinguishing between potentially risky and safe cues. Early learning is the key to avoid mistakes later on, and Ferrari and Chivers (2009a) showed that stimuli experienced by embryonic prey affect their propensity to learn associations with these stimuli later in life. Embryonic woodfrogs, Rana sylvatica, exposed to salamander odour for five consecutive days while in the egg, subsequently failed to recognize salamander cues as risky when a learning task was undertaken with the 2-week-old tadpoles.

Here, we investigate another way through which prey could enhance their ability to distinguish predators from non-predators through latent inhibition. If prey can learn to recognize a novel species as non-threatening, could they use this information to infer the riskiness (or lack thereof) associated with other novel species, based on the similarities to the reference species? The analogous phenomenon of generalization of predator recognition was put forward in a few key studies (Griffin et al. 2001; Ferrari et al. 2007; Stankowich and Coss 2007) demonstrating that some prey species could infer information about unknown novel predators based on the chemical or visual similarities between those predators and cues from predators that they already recognize. However, the key difference between generalizing predators and non-predators is the pay-off resulting from the cost-benefit analysis of the behaviour. We can predict the costs associated with wrong associations in generalization contexts. In the case of generalization of predator recognition, a 'false alarm' (type I error) would be the labelling of a novel non-predatory species as 
threatening, and would result in the prey wasting time and energy in unnecessary predator avoidance, but would not lead to mortality. In the case of generalization of nonpredator recognition, a 'false alarm' would be the labelling of a predatory species as non-threatening. This would presumably increase the probability of capture, and thus mortality risk, of the individual. Consequently, the costs associated with mistakes are much higher in the non-predator generalization context and we could then predict a much narrower frame of generalization, if any, in this context.

In this experiment, we exposed embryonic woodfrogs to the odour of a tiger salamander (Ambystoma tigrinum) for seven consecutive days. This step should theoretically teach woodfrogs to treat tiger salamanders as non-risky. Two weeks post-hatching, tadpoles were subjected to a learning task, being presented with injured conspecific cues paired with the odour of tiger salamander, red-bellied newt (close relative, Cynops pyrrhogaster) or goldfish (Carassius auratus). To investigate if tadpoles have learned to recognize the cues as risky, we measured their antipredator responses when subsequently presented with salamander, newt or fish odour. We predict that the embryonic preexposure to salamander cues will inhibit the learning of salamander cues but should not stop the association between fish cues and risk. If woodfrogs can infer the nonthreatening nature of newts based on having learned that the salamander is not a threat, then we should see inhibition of learning of the newt cues as well.

Throughout our paper, we will continually refer to prey learning to recognize and generalize predators and nonpredators. We do this for convenience; however, the reality is that learning to recognize predator odours from nonpredator odours may actually represent recognizing risky from safe stimuli and not predators and non-predators per se. When the tadpoles learn that the salamander cue is risky, it may reflect that the tadpoles learn that the salamander is risky or that the habitat that contains the salamander is risky. Likewise, if they learn the salamander is non-threatening, then it may mean that the salamander is a non-predator or that the habitat where salamanders are detected is non-threatening. Generalizing the risk associated with salamanders and newts can thus be considered within the framework of generalizing predators versus nonpredators or the framework of generalizing dangerous versus safe habitats.

\section{Materials and methods}

Water and predators

One week prior to starting the experiment, a 1900-L tub (food-grade plastic water trough) was filled with well water and left outdoors. The tub was seeded with zooplankton, phytoplankton and aquatic vegetation (sedges, slough grasses, horsetails) from a local pond using fine mesh dip net. This ensured that the holding and testing water contained a large array of odours but that no salamander cues were present in the water.

Three tiger salamanders (snout-vent length: mean \pm $\mathrm{SD}=90 \pm 6 \mathrm{~mm}$ ) were captured from a pond on the University of Saskatchewan campus using Gee's improved minnow traps. The salamanders were transported to the field site and maintained on a diet of earthworms. Four redbellied newts (snout-vent length: $40 \pm 3 \mathrm{~mm}$ ) and two goldfish (fork length: $71 \pm 5 \mathrm{~mm}$ ) were purchased from a local pet store and were individually housed in plastic containers filled with well water. The newts were fed newt bites (HBH Newt and Salamander Bites, Springville, UT) and goldfish were fed fish flakes (Nutrafin Max Goldfish Flake Food, Rolf C. Hagen Inc., Montreal, QC).

Test subjects and stimulus preparation

All eggs and tadpoles used in this experiment were collected from a single pond in central Alberta in May 2009. Work from past field seasons showed that woodfrogs from this pond do not have innate recognition of salamander predators (Ferrari and Chivers 2009a, b). Red-bellied newts and goldfish do not naturally occur in the region of our field site. Egg laying began on April 28 and was completed on May 11.

Predator odours were prepared prior to the start of the experiment and frozen until needed. Ferrari et al. (2006) showed that prey animals do not have an innate sense of whether the predator odours they learn are concentrated or not. However, they can adjust the intensity of their antipredator responses to changes in relative concentration once they have learned that the predator odour represents a threat. Although we do not know 'how much' odours are produced by each species, we decided to use body volume as a way to approximate odour production among species. Odours were made from one salamander, four newts and two goldfish. To avoid any problem with using the odour of a single salamander, we used three different salamanders to prepare the cues, and randomly assigned the salamander cues throughout the experiment. Odours were obtained from maintaining the live animals (one salamander, four newts or two goldfish) in 21 of well water for $24 \mathrm{~h}$. After this period, the stimulus was bagged and frozen at $-20^{\circ} \mathrm{C}$. The procedure was repeated for four consecutive days. The stimuli were thawed and brought to ambient temperature prior to being used.

Experimental setup

Previous studies have shown maternal effects on antipredator responses of amphibians (Moore et al. 1996). 
Consequently, we controlled for maternal effects in our study by testing eggs from different clutches. Five freshly laid egg clutches, laid the previous night, were collected and each clutch was divided into four sub-clutches of approximately 50-60 eggs each. Male and female wood frogs breed only once a season, hence eggs obtained from different clutches are probably unrelated (Halverson et al. 2006). The 20 sub-clutches were then transferred into individual 3.5-1 pails filled with 31 of conditioned well water. The sub-clutches consisted of a single mass of eggs with the egg jelly intact. Examinations of eggs from all five clutches revealed that they were at the Gosner developmental stage 10-11 (Gosner 1960). At this stage, the neural tube is not yet formed.

\section{Experimental procedure}

\section{Embryonic pre-exposure phase}

Two of the four sub-clutches from each clutch were randomly assigned to one of two treatments: (1) Water: $20 \mathrm{ml}$ of well water, or (2) Salamander odour: $20 \mathrm{ml}$ of salamander odour. Hence, we treated 20 pails, 10 receiving the water treatment and 10 receiving the salamander odour treatment. Eggs were treated daily at $1500 \mathrm{~h}$ for 7 days (day 1-7); the stimuli were slowly injected on the side of the pails to minimize disturbance to the eggs. At $1700 \mathrm{~h}$ each day, a $100 \%$ water change was performed on all the pails. The experimenter was wearing latex gloves to avoid the transfer of any odour to the embryos. Eggs were treated until the embryos within the eggs appeared fully formed (approx. Gosner stage 22/ 24) but had not hatched. We verified that the embryos had not hatched as they were curled up inside their eggshell. Tadpoles straighten out immediately upon hatching. The treatments stopped after day 7. Embryos started hatching the following day, and all the embryos were hatched by the following evening. Tadpoles were provided with rabbit food, and the water was partially changed every second day. Tadpoles were raised for 12 days to Gosner stage 25 .

\section{Larval conditioning phase}

Eighteen tadpoles from each of the 20 pails were arbitrarily chosen and placed individually into $0.5-1$ plastic cups (10 cm diameter, $12 \mathrm{~cm}$ high) filled with well water. Out of the 18 tadpoles, 6 were subsequently exposed to $5 \mathrm{ml}$ of injured conspecific cues paired with $5 \mathrm{ml}$ of salamander odour, six tadpoles were exposed to $5 \mathrm{ml}$ of injured conspecific cues paired with $5 \mathrm{ml}$ of newt odour and six tadpoles were exposed to $5 \mathrm{ml}$ of injured conspecific cues paired with $5 \mathrm{ml}$ of fish odour. The injured conspecific cues were obtained by euthanizing 120 tadpoles with a blow to the head and then immediately crushing them using a mortar and pestle and adding $600 \mathrm{ml}$ of water (in accordance with Animal Care protocol 20060014). One hour after the conditioning phase, a $100 \%$ water change was conducted on all the cups, and food was provided to the tadpoles.

\section{Larval testing phase}

Twenty-four hours after the conditioning phase, tadpoles from each group were tested for their responses to the odour of salamander, newt or fish. Previous studies of this system have shown that tadpoles do not respond to the injection per se, but rather to the odour of species they recognize as dangerous (Ferrari and Chivers 2009a, b, c). For this reason, and to reduce the number of animals used in our experiment, we did not add an additional distilled water treatment. Tadpoles were tested using a well-established protocol (Ferrari and Chivers 2009a, b). The trials consisted of a 4-min pre-stimulus followed by a 4-min post-stimulus injection period during which the behaviour (activity) of the tadpoles was recorded. The two periods were separated by a 30 -sec injection period, during which $10 \mathrm{ml}$ of salamander, newt or goldfish odour was slowly introduced on the side of the cup using a syringe to minimize disturbance. The cues were added to the side of the cup opposite the tadpole. Tadpoles have been shown to decrease activity in response to predation cues. Hence, a line was drawn in the middle of the cup, and the number of line crosses was counted during the two observation periods. We considered that a tadpole crossed a line when its entire body was on the other side of the line. The trials were performed outdoors. The order of the treatments was randomized throughout the day. The observer was blind to the treatments.

\section{Statistical analysis}

No differences in pre-stimulus data were found across treatment (average pre-stimulus line crosses $=25 / 4 \mathrm{~min}$ period; 2-way ANOVA, pre-exposure: $F_{1,393}=0.29$, $P=0.59$; conditioning: $F_{2,393}=0.22, P=0.81$; interaction: $\left.F_{2,393}=1.39, P=0.25\right)$. We calculated the change in proportion of line crosses from the pre-stimulus baseline [(post-pre)/pre]. The data were normally distributed, and the variances were homogeneous among treatments. The data were analysed using a randomized block design (with replication) ANOVA approach, whereby the effects of 'embryonic pre-exposure' (water vs. salamander odour), 'conditioning' (injured cues paired with the odour of salamander, newt or fish) and 'testing cue' (salamander, newt or fish odour) were treated as fixed factors, whereas 
'clutch' was included as a random factor (blocking factor), to account for potential maternal effects. While conditioning and testing trials were performed on individual tadpoles, tadpoles raised in the same pail received the same embryonic pre-exposure treatment, and hence cannot be considered independent. To account for data dependency, we averaged the data obtained from tadpoles raised from the same pail, receiving the same conditioning treatment and tested for the same cue. Hence, pail, not tadpole, was used as our sampling unit. Due to significant interactions, the effects of testing cues were assessed for each embryonic exposure and conditioning cue combination. Post hoc Tukey comparisons were performed to compare the responses to the three predator cues, if significantly different.

\section{Results}

The overall 3-way ANOVA revealed a significant 3-way interaction between pre-exposure cues, conditioning cues and testing cues $\left(F_{4}, 16.3=5.1, P=0.004\right)$ and no effect of clutch $(P=0.73$, all $P>0.1$ in 2 - and 3 -way interactions). These results support previous work showing that 'clutch' was never found to have an effect of the responses to predators in our system (Ferrari and Chivers 2009a, b, c). Hence, we removed 'clutch' and performed individual 2-way ANOVAs and subsequent one-way ANOVAs for each of the pre-exposure groups to investigate the nature of this interaction.

\section{Woodfrogs pre-exposed to water as embryos}

A statistically significant interaction was found between conditioning and testing cues $\left(F_{4,79}=23.8, P<0.001\right.$; Fig. 1, top panel), indicating that the responses of tadpoles to the predator odour were dependent on the conditioning treatment received. Tadpoles conditioned to recognize salamander (TP + Salamander) responded with a different intensity to the three cues $\left(F_{2,29}=34.8, P<0.001\right)$, displaying their strongest antipredator response to salamander odour, a weaker one to the closely related newt odour, but did not show a consistent reduction in activity in response to fish odour. Tadpoles conditioned to recognize newt odour $(\mathrm{TP}+\mathrm{Newt})$ responded with a similar pattern $\left(F_{2,30}=13.3, \quad P<0.001\right)$, however, displaying their strongest antipredator response to newt odour, a weaker one to salamander odour and no consistent reduction in activity in response to the fish odour. Tadpoles conditioned to recognize the fish $(\mathrm{TP}+$ Fish) displayed a strong antipredator response to fish odour only $\left(F_{2,29}=12.0\right.$, $P<0.001)$, but did not reduce activity to salamander or newt odour (Fig. 1, top panel).

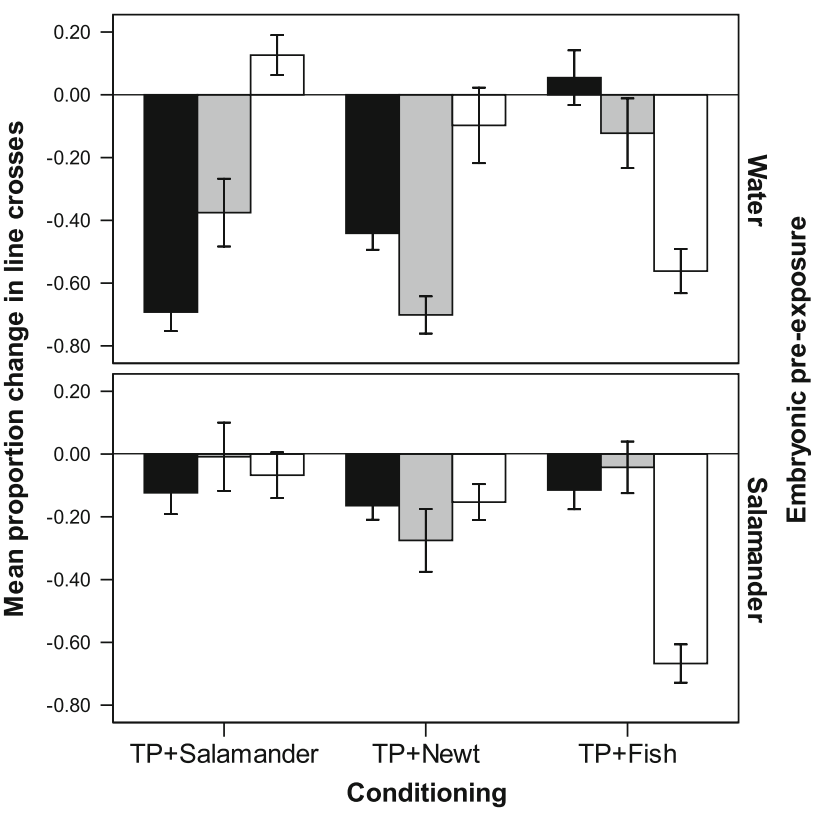

Fig. 1 Mean $( \pm S E)$ proportion change in line crosses from the prestimulus baseline for woodfrog tadpoles exposed to salamander odour (black bars), newt odour (grey bars) or fish odour (white bars), 1 day after being conditioned with crushed tadpoles paired with salamander odour (TP + Salamander), newt odour $(\mathrm{TP}+\mathrm{Newt})$ or fish odour $(\mathrm{TP}+\mathrm{Fish})$. Embryonic woodfrogs were exposed to water or salamander odour for 6 days

Woodfrogs pre-exposed to salamander as embryos

A statistically significant interaction was also found between conditioning and testing cues $\left(F_{4,77}=9.1\right.$, $P<0.001$; Fig. 1, bottom panel), indicating that the responses of tadpoles to the predator odour were dependent on the conditioning treatment received. Tadpoles conditioned to fish odour responded with a stronger intensity to fish odour than salamander or newt odour $\left(F_{2,29}=31.1\right.$, $P<0.001$, Fig. 1), with no difference in the responses of tadpoles to newt or salamander odour (Tukey HSD: $P=0.83$ ). In contrast, tadpoles conditioned with salamander odour or newt odour did not respond differently to the three odours $\left(F_{2,29}=0.04, P=0.92\right.$ and $F_{2,28}=0.98$, $P=0.44$, respectively).

\section{Discussion}

Our results indicate that amphibians have the ability to collect information about predatory and non-predatory species present in their vicinity during the embryonic stage and use this information at later ontogenetic stages to infer on the potential riskiness of novel species present in their environment. Embryonic woodfrogs exposed to the water control subsequently learn to identify any novel predator cues paired with injured conspecific cues as risky. 
Moreover, they were able to generalize their recognition from a salamander to a novel newt and from a newt to a novel salamander. When tadpoles recognized fish cues as risky, they did not generalize their recognition to distantly related salamanders or newts. These results concur with those obtained previously on larval and embryonic amphibians (Ferrari and Chivers 2009c). When embryonic woodfrogs were exposed to salamander odour, tadpoles subsequently failed to learn to recognize either salamander odour or newt odour as potential risky stimuli, demonstrating the ability of tadpoles to use embryonic information and infer on the potential risk of non-predators. However, salamander odour pre-exposure did not alter the ability of tadpoles to learn to recognize fish odour as risky.

We do not know whether the learned recognition and generalization we documented represents recognition of predators or non-predators per se, or whether it represents a more general recognition of dangerous versus safe cues. If the embryos were learning that an area that contains salamander odour was dangerous, then they may be able to generalize this information to other similar habitats, for example, those that smell of newts. Likewise, if they learned that an area containing salamander cues was not dangerous, then they may be able to generalize this information to other similar smelling habitats. Chivers and Smith $(1995 \mathrm{a}, \mathrm{b})$ showed that minnows conditioned to recognize water from a habitat that was vegetated with emergent cattails (Typha latifotia) and bulrushes (Scirpus spp.) did not generalize their recognition to water from an open habitat located $5 \mathrm{~m}$ from the nearest vegetation. Given that these two sites represent extremes of the habitat types in the creek, we should not expect generalization to occur. However, if the sites were more similar (i.e. differed in the density or species composition of plants) then we may expect that prey could exhibit generalization of dangerous and safe habitats. This is an exciting area of future work. Prey are known to exhibit strong preference for specific habitats, however, the role of learning and generalization in such preferences has received little attention (Lima and Dill 1990; Lima 1998). There is some evidence that embryonic pre-exposure of amphibians to predation risk can alter subsequent habitat use. Mathis et al. (2008) exposed embryonic ringed salamanders (Ambystoma annulatum) to potential predators and found that hatchling larvae show a preference for vegetated habitats, an area where their risk of predation is lower. Exposure of embryos to non-predators and blank controls did not cause an alteration of habitat preferences of the hatchling larvae.

How does non-predator generalization fit in the 'risk paradigm' used by prey animals? Our results indicate that the frame of generalization of non-predator information is at least as wide, if not wider, than the frame of generalization of predator recognition. When a prey generalizes its recognition from a known predator to a novel species, the intensity of its antipredator response is not as strong as the intensity of response to the known predator. In our experiment, woodfrogs learning to recognize salamanders or newts as risky responded to the other predator with a lower intensity of antipredator response (Fig. 1, top panel). Ferrari et al. (2007) likewise showed that the perception of risk by prey fish was inversely related to the degree of similarity between the known predator and the novel species. These weaker responses do not reflect the prey's knowledge that its chances of escaping the predator are higher, but rather that the probability of attack is lower, due to the increased uncertainty that the novel species is indeed a predator. In the context of non-predator generalization, a parallel outcome would have been reached if the preexposure to the known non-predator resulted in an antipredator response to the closely related species, in the group trained to recognize the closely related species as predatory. In other words, tadpoles pre-exposed to salamander odour and conditioned to recognize newts as a predator would be predicted to show an antipredator response to newts, although lower than the response exhibited by their non-pre-exposed counterparts. The fact that the pre-exposure to a non-predator entirely suppressed the formation of even mild risk recognition of the new species is puzzling. Although not significant, there may be a trend of the tadpoles to respond with a weak intensity to newts (Fig. 1, bottom panel).

To fully understand the consequence of such risk-overestimation or risk-underestimation, we must first understand the impact of such decisions for the processing of the next piece of information and more specifically consider the amount of additional information required to rectify erroneous information. For the purpose of clarity, we used the term 'risk-underestimation' to describe behaviours or strategies that are less risk-aversive than the 'risk-overestimation' ones and are not referring to the two extremes of a 'risk' continuum. A few studies have looked at the rule of thumb used by prey to deal with conflicting cues regarding the level of threat of predators. It takes only one piece of information to increase the perceived risk associated with a predator, but it takes many successive and consistent pieces of information to decrease the perceived risk associated with a predator (Ferrari and Chivers 2006, 2009b). This phenomenon is consistent with risk-overestimation decision-making, whereby the probability of over-estimating the risk associated with a predator is often higher than the probability of under-estimating the risk associated with a predator (Bouskila and Blumstein 1992). However, this asymmetry may counter-balance the apparently risk-prone nature of non-predator generalization.

Another factor to consider is the probability of finding non-predatory species and closely related predatory species 
in sympatry. It may be possible for different prey species to show different patterns of responses to predatory and nonpredatory species according to the evolutionary history of those species with specific predator communities. In communities where closely related predators and non-predators are rare or non-existent, the probability of a novel species being predatory given that a closely related species is not will be lower than in communities where closely related predators and non-predators are common. Hence, evolutionary history should be able to shape this 'prior' distribution, effectively increasing or decreasing the chances of 'false alarm' and thus affecting the amount or strength of generalization observed.

One of the main factors affecting the propensity of some individuals to under or over-estimate risk is state dependency. One interesting avenue to pursue is the role of state-dependence on the predator and non-predator generalization frame of prey species. This concept can also be extended beyond intraspecific state-dependent comparisons to interspecific life-history strategies. Larval amphibians might be predisposed to exhibit over-estimation of risk because of the temporal and energetic constraints related to their life history. They may find themselves in a race against time, in which resource gathering and high growth rate are keys to allow metamorphosis before their habitat dries up. Even if they metamorphose prior to pond drying, smaller size at metamorphosis may lead to lower overwinter survival (Smith 1987). Comparing the generalization frame of species with different life histories will give us insights into factors affecting both qualitative and quantitative information processing.

Learning to recognize risk associated with alarm cues appears to be a nearly universal form of predator learning for aquatic animals (e.g. amphibians: Woody and Mathis 1998; Mirza et al. 2006; fishes: Mirza and Chivers 2000; gastropods: Rochette et al. 1998; insects: Wisenden et al. 1997; flatworms: Wisenden and Millard 2001). The only time this mode of learning has been shown to fail is when the prey is pre-exposed to the odour prior to conditioning with alarm cues. Aside from this study, latent inhibition has been shown in fishes (Ferrari et al. 2006) and crayfishes (Acquistapace et al. 2003; Hazlett 2003), indicating that this ability is not limited to vertebrates and may in fact be widespread. However, one can wonder about the adaptive value of latent inhibition if the non-predator learned at one life stage becomes predatory for the next life stage. In our case, the salamander may not be a predator of woodfrog eggs, but is certainly a threat to tadpoles, and embryonic learning of salamanders as non-predatory may be quite maladaptive for tadpoles. This and previous studies (Mathis et al. 2008, Ferrari and Chivers 2009a) have shown that embryos can learn to recognize predators if the predator odour is paired with the odour of crushed conspecifics of another life stage (i.e., injured tadpole cues). Hence, a tadpole predator will not be recognized as non-predatory by the embryos if this predator is actively foraging on tadpoles, and releasing injured tadpole cues either through direct consumption or via diet cues in the water column (Chivers and Mirza 2001). In the event that embryos inadvertently learn a species as non-predatory when in fact it is a predator, multiple exposures to the predator odour paired with injured conspecific cues would override the latent inhibition effects (Mitchell, McCormick, Ferrari and Chivers, unpublished data). The next logical step for researchers attempting to identify how prey learn to distinguish predators from non-predators is to ask: what happens when the animal is given conflicting information through time? Foster and Kokko (2009) provide a great model for us to consider these questions. Even more interesting would be to determine if there is an asymmetry in the amount of information needed to overcome predator learning vs non-predator learning.

Acknowledgments We thank Glen and Jean Chivers for letting us invade their home and wetlands. Thanks to Aditya Manek and Candace Piper for trapping the salamanders, and Aditya Manek and Oliver for field assistance. This research was funded through an NSERC Discovery Grant to DPC. All work reported therein was in accordance with the Canadian Council on Animal Care and followed the University of Saskatchewan Animal Care Protocol no. 20070014.

Open Access This article is distributed under the terms of the Creative Commons Attribution Noncommercial License which permits any noncommercial use, distribution, and reproduction in any medium, provided the original author(s) and source are credited.

\section{References}

Acquistapace P, Hazlett BA, Gherardi F (2003) Unsuccessful predation and learning of predator cues by crayfish. J Crust Biol 23:364-370

Bouskila A, Blumstein DT (1992) Rules of thumbs of predation hazard assessment: predictions from a dynamic model. Am Nat 139:161-176

Brown GE, Chivers DP (2005) Learning as an adaptive response to predation. In: Barbosa P, Castellanos I (eds) Ecology of predator/ prey interactions. Oxford University Press, Oxford, pp 34-54

Chivers DP, Mirza RS (2001) The importance of predator-diet cues in the responses of larval woodfrogs to fish and invertebrate predators. J Chem Ecol 27:45-51

Chivers DP, Smith RJF (1995a) Fathead minnows (Pimephales promelas) learn to recognize chemical stimuli from high-risk habitats by the presence of alarm substance. Behav Ecol 6:155-158

Chivers DP, Smith RJF (1995b) Chemical recognition of risky habitats is culturally transmitted among fathead minnows, Pimephales promelas (Osteichthyes, Cyprinidae). Ethology 99:286-296

Curio E (1988) Cultural transmission of enemy recognition by birds. In: Zentall TR, Galef BG Jr (eds) Social learning: psychological and biological perspectives. Hillsdale, New Jersey, pp 75-97 
Dall SRX, Giraldeau L-A, Olsson O, McNamara JM, Stephens DW (2005) Information and its use by animals in evolutionary ecology. Trends Ecol Evol 20:187-193

Ferrari MCO, Chivers DP (2006) The role of latent inhibition in acquired predator recognition by fathead minnows. Can J Zool 84:505-509

Ferrari MCO, Chivers DP (2009a) Latent inhibition of predator recognition by embryonic amphibians. Biol Lett 5:160-162

Ferrari MCO, Chivers DP (2009b) Temporal variability, threatsensitivity and conflicting information about the nature of risk: understanding the dynamics of tadpole antipredator behaviour. Anim Behav 78:11-16

Ferrari MCO, Chivers DP (2009c) Sophisticated early life lessons: generalization of predator recognition by frog embryos. Behav Ecol 20:1295-1298

Ferrari MCO, Kapitania-Kwok T, Chivers DP (2006) The role of learning in the development of threat-sensitive predator avoidance: the use of predator cue concentration by fathead minnows. Behav Ecol Sociobiol 60:522-527

Ferrari MCO, Gonzalo A, Messier F, Chivers DP (2007) Generalization of learned predator recognition: an experimental test and framework for future studies. Proc R Soc B 274:1853-1859

Ferrari MCO, Wisenden BD, Chivers DP (2010) Chemical ecology of predator-prey interactions in aquatic ecosystems: a review and prospectus. Can J Zool 88:698-724

Foster KR, Kokko H (2009) The evolution of superstitious and superstition-like behaviour. Proc R Soc B 276:31-37

Galef BJ Jr, Laland KN (2005) Social learning in animals: empirical studies and theoretical models. Bioscience 55:489-499

Gosner KL (1960) A simplified table for staging anuran embryos and larvae with notes on identification. Herpetologica 16:183-190

Griffin AS (2004) Social learning about predators: a review and prospectus. Learn Behav 32:131-140

Griffin AS, Evans CS, Blumstein DT (2001) Learning specificity in acquired predator recognition. Anim Behav 62:577-589

Halverson MA, Skelly DK, Caccone A (2006) Inbreeding linked to amphibian survival in the wild but not in the laboratory. J Hered 97:499-507

Hazlett BA (2003) Predator recognition and learned irrelevance in the crayfish Orconectes virilis. Ethology 109:765-780

Lima SL (1998) Nonlethal effects in the ecology of predator-prey interactions-what are the ecological effects of anti-predator decision-making? Bioscience 48:25-34
Lima SL, Dill LM (1990) Behavioral decisions made under the risk of predation: a review and prospectus. Can J Zool 68:619-640

Mathis A, Chivers DP, Smith RJF (1996) Cultural transmission of predator recognition in fishes: intraspecific and interspecific learning. Anim Behav 51:185-201

Mathis A, Ferrari MCO, Windel N, Messier F, Chivers DP (2008) Learning by embryos and the ghost of predation future. Proc R Soc B 275:2603-2607

Mirza RS, Chivers DP (2000) Predator-training enhances survival of brook trout: evidence from laboratory and field enclosure studies. Can J Zool 78:2198-2208

Mirza RS, Ferrari MCO, Kiesecker JM, Chivers DP (2006) Responses of American toad tadpoles to predation cues: behavioural response thresholds, threat-sensitivity and acquired predation recognition. Behaviour 143:887-889

Moore RD, Newton B, Sih A (1996) Delayed hatching as a response of streamside salamander eggs to chemical cues from predatory sunfish. Oikos 77:331-335

Rochette R, Arsenault DJ, Justome B, Himmelman JH (1998) Chemically-mediated predator-recognition learning in a marine gastropod. Ecoscience 5:353-360

Sih A (1992) Prey uncertainty and the balancing of antipredator and feeding needs. Am Nat 139:1052-1069

Smith DC (1987) Adult recruitment in chorus frogs: effects of size and date at metamorphosis. Ecology 68:344-350

Stankowich T, Coss RG (2007) The re-emergence of felid camouflage with the decay of predator recognition in deer under relaxed selection. Proc R Soc B 274:175-182

Wisenden BD, Harter KR (2001) Motion, not shape, facilitates association of predation risk with novel objects by fathead minnows (Pimephales promelas). Ethology 107:357-364

Wisenden BD, Millard MC (2001) Aquatic flatworms use chemical cues from injured conspecifics to assess predation risk and to associate risk with novel cues. Anim Behav 62:761-766

Wisenden BD, Chivers DP, Smith RJF (1997) Learned recognition of predation risk by Enallagma damselfly larvae (Odonata, Zygoptera) on the basis of chemical cues. J Chem Ecol 23: 137-151

Woody DR, Mathis A (1998) Acquired recognition of chemical stimuli from an unfamiliar predator: associative learning by adult newts, Notophthalmus viridescens. Copeia 1998:1027-1031 